\title{
A formalization of one of the main claims of "ALS-implicated protein TDP-43 sustains levels of STMN2, a mediator of motor neuron growth and repair" by Klim et al. $2019^{1}$
}

\section{B. Nolan Nichols}

Maze Therapeutics, USA

E-mails:nnichols@mazetx.com,nolan.nichols@gmail.com; ORCID:

https://orcid.org/0000-0003-1099-3328

Editor: Cristina-Iulia Bucur (https://orcid.org/0000-0002-7114-6459)

Review comments from: Tobias Kuhn (https://orcid.org/0000-0002-1267-0234); Davide Ceolin

(https://orcid.org/0000-0002-3357-9130); Cristina-Iulia Bucur (https://orcid.org/0000-0002-7114-6459)

Received 15 October 2021

Accepted 12 November 2021

\begin{abstract}
Klim et al. claimed in previous work that the protein TDP-43 generally contributes to the transcription of STMN2 in human motor neurons. We present here a formalization of that claim, stating that all things of class "TAR DNA binding protein" that are in the context of a thing of class "human motor neuron" can generally have a relation of type "contributes to" to a thing of class "transcription of stmn2" in the same context.
\end{abstract}

Keywords: Human motor neuron, TAR DNA binding protein, transcription of stmn2

\section{Introduction}

Klim et al. [2] state that "expression of STMN2, which encodes a microtubule regulator, declined after TDP-43 knockdown and TDP-43 mislocalization as well as in patient-specific motor neurons". We present here a formalization of the main scientific claim from this quote by using a semantic template called the super-pattern [1].

\footnotetext{
${ }^{1}$ As RDF/nanopublication: http://purl.org/np/RAmG2bXxwkIzARk4Mda-lqZU0RVnkpX7hUHBIPcdLHQUU 


\section{Formalization}

Our formalization looks as follows:

$\begin{array}{ll}\text { CONTEXT-CLASS (“in the context of all ...”): } & \text { human motor neuron } \\ \text { SUBJECT-CLASS (“things of type ...”): } & \text { TAR DNA binding protein } \\ \text { QUALIFIER: } & \text { can generally } \\ \text { RELATION-TYPE (“have a relation of type...”): } & \text { contributes to } \\ \text { OBJECT-CLASS (“to things of type...”): } & \text { transcription of stmn2 }\end{array}$

In the context class we use the class "human motor neuron" (Q101404862) from Wikidata. In the subject class, we use the class "TAR DNA binding protein" (Q21133247) from Wikidata. In the object class we minted a new class "transcription of stmn2" that is a subclass of "transcription" (Q177900) from Wikidata and is related to the class "STMN2" (Q18036104) from Wikidata.

\section{RDF code}

This is our formalization as a nanopublication in TriG format:

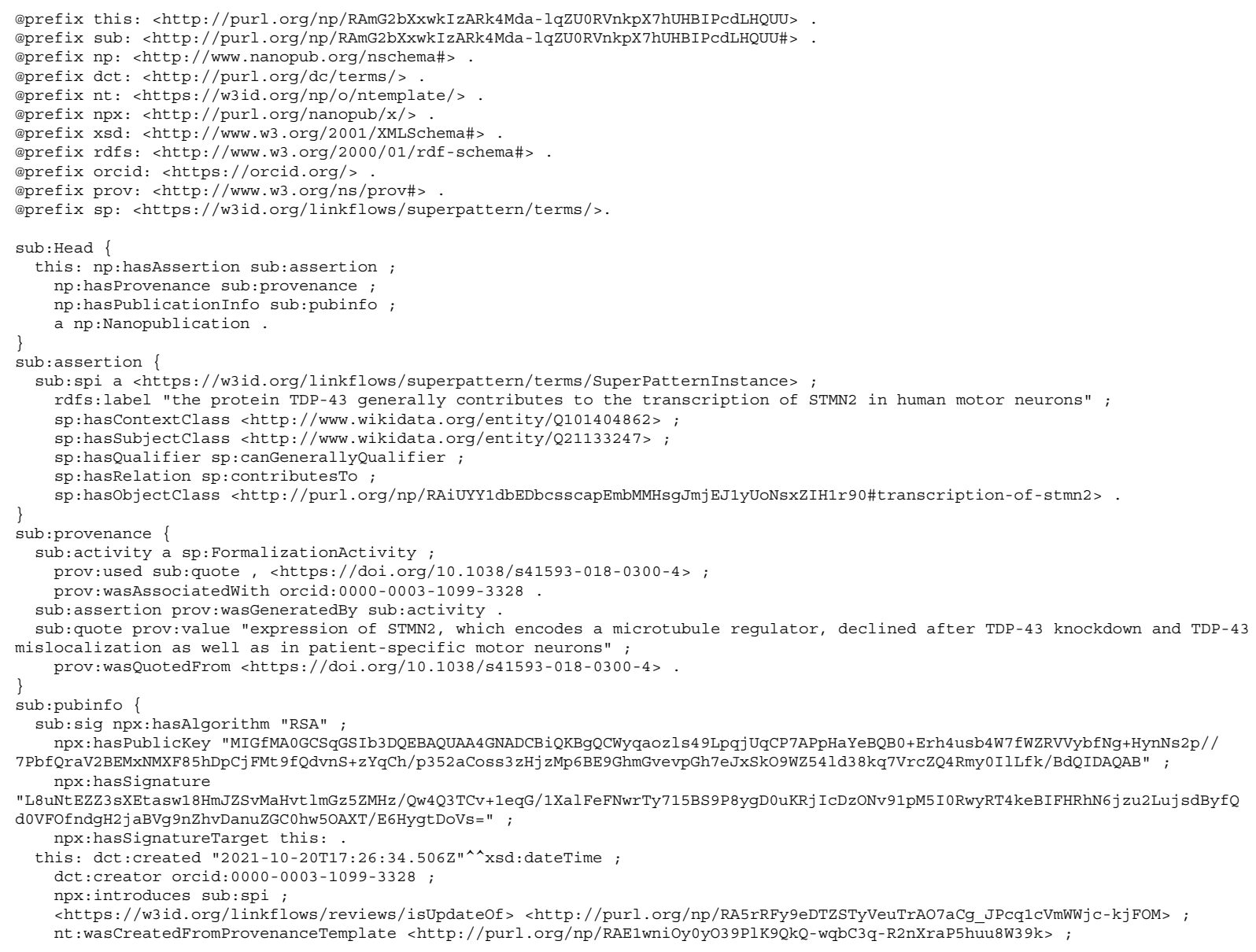


nt: wasCreatedFromPubinfoTemplate <http://purl.org/np/RAA2MfqdBCzmz9yVWj KLXNbyfBNCwSMmOqCNUxkk1maIM> , <http://purl.org/np/RAOGu9Lh0BD4tbIRB9RG6RGRA_ObDh75NTbIqaWgxxs8M>

nt: wasCreatedFromTemplate <http://purl.org/np/RAv68imZrEjfcp2rnEg1hzoBqEVc0coMtp9_1Za0BxNM4> .

The following nanopublications introduce the newly minted classes in TriG format.

This is the class definition of "transcription of stmn2":

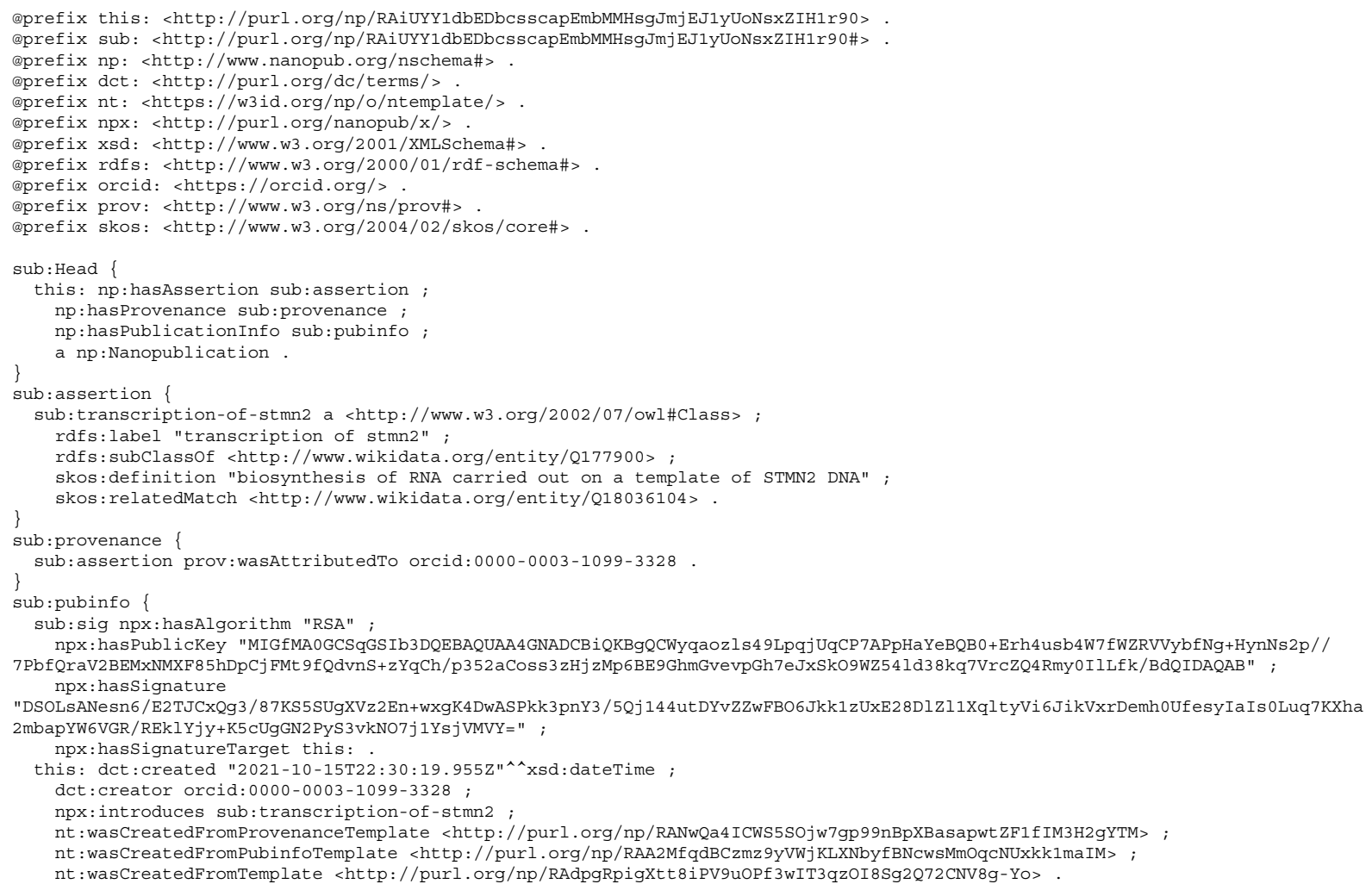

\section{References}

[1] C.I. Bucur, T. Kuhn, D. Ceolin and J. van Ossenbruggen, Expressing high-level scientific claims with formal semantics, in: Proceedings of the 11th Knowledge Capture Conference 2021. doi:10.1145/3460210.3493561.

[2] J.R. Klim, L.A. Williams, F. Limone et al., ALS-implicated protein TDP-43 sustains levels of STMN2, a mediator of motor neuron growth and repair, Nat Neurosci 22 (2019), 167-179. doi:10.1038/s41593-018-0300-4. 